\title{
BAYESIAN PORTFOLIO SELECTION WITH MULTIVARIATE RANDOM VARIANCE MODELS
}

\author{
Refik Soyer* \\ Department of Management Science \\ The George Washington University \\ Washington, DC, U.S.A. \\ Kadir Tanyeri \\ Department of Engineering Management and Systems Engineering \\ The George Washington University \\ Washington, DC, U.S.A.
}

${ }^{*}$ Corresponding author: Department of Management Science, Monroe Hall 403, The George Washington University, 2115 G Street, NW, Washington, DC 20052, USA. E-mail: soyer@gwu.edu, Tel: +1 (202)994 6445, Fax:+1 (202) 9944930. 


\begin{abstract}
We consider multiperiod portfolio selection problems for a decision maker with a specified utility function when the variance of security returns is described by a discrete time stochastic model. The solution of these problems involves a dynamic programming formulation and backward induction. We present a simulation-based method to solve these problems adopting an approach which replaces the preposterior analysis by a surface fitting based optimization approach. We provide examples to illustrate the implementation of our approach.
\end{abstract}

Key words: Portfolio optimization; Bayesian inference; decision analysis; stochastic volatility; dynamic programming. 


\section{Introduction and Overview}

The portfolio selection problem was originally considered by Markowitz (1952) for an investor who has a sum of money to allocate among $K$ securities. Markowitz's approach to the problem consisted of two steps. First forming a set of efficient portfolios and secondly selecting from the efficient set the one portfolio that provides the investor with the most suitable combination of risk and return. Extension of the Markowitz model was considered by others where particular objective functions were suggested and mean-variance analysis was introduced [see for example, Farrar (1962)].

An earlier decision theoretic approach to the problem was considered by Mao and Sarndal (1966). The decision theoretic point of view provided a justification for the mean-variance analysis. For example, it can be shown that the formulation of Farrar (1962) follows from maximization of expected utility when security returns are normally distributed and the investor's utility function is exponential. Most of the early work in portfolio selection focused on single-period models. Some exceptions to this are the multiperiod models of Mossin (1968).

A Bayesian decision theoretic approach to the problem (with revision of uncertainty) was presented in Winkler and Barry (1975) and Barry and Winkler (1976) where Bayesian multiperiod models were introduced and computational difficulties were pointed out. More recent Bayesian work includes Quintana (1992), Quintana and Putnam (1996) where single period adaptive Bayesian models were considered. In other words, these models used sequential updating and one-step ahead portfolio allocations. Polson and Tew (2000) also presented a single period adaptive Bayesian model using a hierarchical model setup. None of these models considered a formal treatment of stochastic volatility. The recent work by Aguilar and West (2000) introduced single period dynamic factor models with stochastic volatility.

Quintana and Putnam (1994) and Quintana et. al. (1998) considered the multiperiod problem and discussed the difficulties in solving the stochastic dynamic program. In Quintana and Putnam (1994), the authors showed that if the utility function is additive over time, then the optimal solution to the multi-period problem consists of one-period optimal solutions over time. In other words, the one-step look ahead rule is optimal in this case. A comprehensive review of Bayesian work in portfolio management is given in Quintana et. al. (2003).

In this paper we will consider the multi-period portfolio selection problem from a Bayesian decision theoretic point of view. In our development we assume that the security returns are described by multivariate stochastic variance models and in so doing consider both the multivariate GARCH models of Bollerslev et. al. (1988) and 
state-space type stochasic volatilility models of Uhlig (1997) and Liu (2000). We note that the presented approach is general and can be applied to other types of multivariate stochastic volatility models considered by Harvey et. al. (1994) and Soyer and Tanyeri (2003). Our formulation of the problem will follow a setup similar to that considered in Winkler and Barry (1975). We present a solution to the decision problem based on Monte Carlo methods that alleviate some of the difficulties. In section 2 we present the decision theoretic setup for the portfolio selection problem. We focus on the twoperiod problem with stationary returns and show the difficulties involved in the preposterior analysis. In section 3 present we consider the extension of the problem where the returns following two different classes of multivariate stochastic variance models. We introduce a standard Monte Carlo approach to solve the two-period portfolio selection problem in the multivariate stochastic variance models in Section 4 and illustrate the computational burden and inefficiencies involved in implementation of the standard Monte Carlo method. To alleviate these problems we present an alternative Monte Carlo method. Our method is based on the curve/surface fitting approach of Muller and Parmigiani (1995) that was developed for optimal design problems. This approach enables us to perform the preposterior analysis in an efficient manner. An implementation of this approach is illustrated in Section 5 using the two stochastic variance models and the results are compared with the standard Monte Carlo method.

We note that the multi-period allocation problem that we consider here arises also in derivatives pricing in the context of discrete time hedging problem. As pointed out by one of the referees, these problems typically involve allocation between two assets and thus are well-suited to the proposed method.

\section{Decision Theoretic Setup for the Portfolio Selection Problem}

Let $W_{t}$ denote the wealth of the investor at the end of time period $t$ and $W_{0}$ denote the initial wealth of the investor at time 0 . Also, we define $r_{t}^{k}$ as the return from security $k$ during time period $t$. If we assume that there are no transaction costs and the portfolio will be chosen from $K$ securities, then the investor's wealth at the end of period $t$ is given by

$$
W_{t}=\sum_{k=1}^{K} W_{t}^{k}
$$

where $W_{t}^{k}$ is the amount invested in security $k$ at the beginning of time period $t+1$. The wealth of the investor at the end of time period $(t+1)$ can be written as 


$$
W_{t+1}=\sum_{k=1}^{K}\left(1+r_{t+1}^{k}\right) W_{t}^{k}=W_{t}+\sum_{k=1}^{K} r_{t+1}^{k} W_{t}^{k}
$$

Following the development of Winkler and Barry (1975), we assume that the investor's objective is to maximize the utility of wealth at the end of a finite time period $T$, that is, to maximize $U\left(W_{T}\right)$. The form of the utility function describes the investor's attitude towards risk and provides the investor with a combination of risk and return that reflects this attitude. As pointed out by Mao and Sarndal (1966), in finance literature various suggestions have been made to choose a portfolio and these strategies can be justified by specifying different utility functions. For example, the mean-variance analysis is justified by a quadratic utility function irrespective of the distribution of security returns. Another form of mean-variance analysis, as in Farrar (1962), is justified by an exponential utility function when security returns are normally distributed.

In the multiperiod problem with a finite horizon $T$, the investor will be maximizing the utility $U\left(W_{T}\right)$ by sequentially choosing the decision variables $W_{t}^{k}$, $t=0, \ldots, T-1$ and $k=1, \ldots, K$ at different points in time based on the available information. That is, the optimal allocation will be revised as the random quantities $r_{t}^{k}, t=1, \ldots, T$, are observed over time. The multiperiod optimal allocation problem can be represented as a sequential decision problem as given by the $T$-stage decision tree in Figure 1.

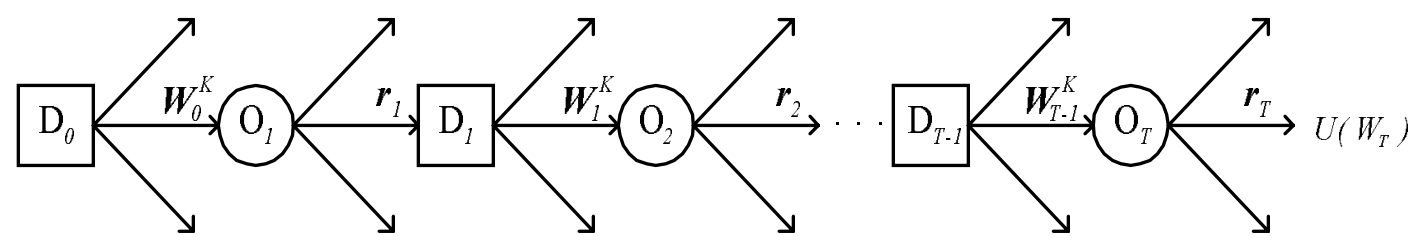

Figure 1. Decision tree for the multiperiod portfolio selection problem.

In Figure 1 the decision nodes at time periods $t=0, \ldots, T-1$ are denoted by $D_{0}, D_{1}, \ldots, D_{T-1}$ and the random (observation) nodes are denoted by $O_{1}, O_{2}, \ldots$, $O_{T}$. At $t=0$, given the initial wealth $W_{0}$, at decision node $D_{0}$, the investor will determine $W_{0}^{K}=\left(W_{0}^{1}, \ldots, W_{0}^{K}\right)$, that is, the amounts invested in the $K$ securities. At time period $t=1$, the returns from $K$ securities, $\boldsymbol{r}_{1}=\left(r_{1}^{1}, r_{1}^{2}, \ldots, r_{1}^{K}\right)^{\prime}$ are observed. This is denoted by the random node $O_{1}$ in the decision tree. Next, given the observed returns $\boldsymbol{r}_{1}$, at decision node $D_{1}$ the investor will determine $W_{1}^{K}=\left(W_{1}^{1}, \ldots\right.$, 
$\left.W_{1}^{K}\right)^{\prime}$ and this process is repeated at the subsequent nodes. As pointed out by Winkler and Barry (1975), the solution of this problem involves a dynamic programming formulation and backward induction. Solution of the tree proceeds in the usual way by taking expectation at random nodes and maximizing the expected utility at the decision nodes [see Lindley (1985)]. At decision node $(T-1)$, given the observed returns $r_{s}^{k}$, $s=1, \ldots, T-1$ and $k=1, \ldots, K$, the decision variables $W_{T-1}^{K}=\left(W_{T-1}^{1}, W_{T-1}^{2}\right.$, $\ldots,\left(W_{T-1}^{K}\right)^{\prime}$ are chosen such that

$$
\max _{W_{T-1}^{K}} E_{T-1}\left[U\left(W_{T}\right)\right]
$$

where $E_{T-1}$ denotes the expectation conditional on the returns from the first $(T-1)$ periods. In other words, the expectation $E_{T-1}$ is taken with respect to the probability distribution of $\boldsymbol{r}_{T}=\left(r_{T}^{1}, r_{T}^{2}, \ldots, r_{T}^{K^{\prime}}\right)^{\prime}$ given $\mathbf{D}_{T-1}=\left(\boldsymbol{r}_{1}, \boldsymbol{r}_{2}, \ldots, \boldsymbol{r}_{T-1}\right)$. We will denote this distribution by $p\left(\boldsymbol{r}_{T} \mid \mathbf{D}_{T-1}\right)$ assuming all of its parameters are known.

Let $U_{T-1}^{*}\left(W_{T}\right)$ denote the optimal value of the expected utility corresponding to the optimal allocation $\boldsymbol{W}_{T-1}^{K *}=\left(W_{T-1}^{1 *}, W_{T-1}^{2 *}, \ldots, W_{T-1}^{K *}\right)$ at time $(T-1)$. At time $(T-2)$, that is, at decision node $(T-2)$, the optimal allocation is obtained via

$$
\max _{\boldsymbol{W}_{T-2}^{\boldsymbol{K}}} E_{T-2}\left[U_{T-1}^{*}\left(W_{T}\right)\right]
$$

where $U_{T-1}^{*}\left(W_{T}\right)=E_{T-1}\left[U\left(W_{T-1}+\sum_{k=1}^{K} r_{T}^{k} W_{T-1}^{k *}\right)\right]$. In general at time period $t$, given the observed returns $\mathbf{D}_{t}$ the decision variables $W_{t}^{K}=\left(W_{t}^{1}, W_{t}^{2}, \ldots, W_{t}^{K}\right)^{\prime}$ are chosen such that

$$
\max _{\boldsymbol{W}_{\boldsymbol{t}}^{\boldsymbol{K}}} \quad E_{t}\left[U_{t+1}^{*}\left(W_{T}\right)\right]
$$

where $E_{t}$ denotes the expectation conditional on the returns $\boldsymbol{r}^{(t)}$ from the first $t$ periods and $U_{t+1}^{*}\left(W_{T}\right)$ is the expected utility corresponding to the optimal allocation at time periods $t+1, \ldots, T-1$. In this case expectation, $E_{t}$, is taken with respect to the probability distribution $p\left(\boldsymbol{r}_{t+1} \mid \mathbf{D}_{t}\right)$.

Continuing in this manner, at time 0 , the optimal allocation for investment period 1 , is obtained by solving

$$
\max _{W_{0}^{K}} \quad E_{0}\left[\begin{array}{cc}
\max _{W_{1}^{K}} & E_{1}\left[\ldots \max _{W_{T-1}^{K}}\right. \\
\left.\left.E_{t}\left[U\left(W_{T}\right)\right] \ldots\right]\right]
\end{array}\right.
$$


Note that (2.6) is equivalent to solving

$$
\max _{W_{0}^{K}} \quad E_{0}\left[U_{1}^{*}\left(W_{T}\right)\right]
$$

and for the special case of a single period problem, that is, $T=1$, the above reduces to

$$
\max _{W_{0}^{K}}^{K} \quad E_{0}\left[U\left(W_{1}\right)\right]
$$

In general, even for the case of a finite horizon problem, calculation of the optimal allocation is not trivial as it involves implicit computation of expectations and maximizations at each time period. Also, the Bayesian strategy requires revision of uncertainty about the parameters of the distribution of $\boldsymbol{r}_{t}$ after each time period. Thus, the evaluation of the expectations may not be analytically tractable. If $T=1$, then we have a single period problem implying that the investor follows a myopic policy which requires only looking at one period ahead. In this case solution can be obtained for some simple cases.

In what follows we will consider a two-period problem with a specific form for the utility function as well as for the probability distribution of the returns.

\subsection{A Two-period Problem}

To illustrate an implementation of the Bayesian decision theoretic setup now we consider a two-period portfolio selection problem where the investor needs to determine the optimal portfolio so that the utility of wealth at the end of period 2, that is,

$$
U\left(W_{2}\right)=U\left(W_{1}+\sum_{k=1}^{K} r_{2}^{k} W_{1}^{k}\right)=U\left(W_{1}+\boldsymbol{r}_{2}^{\prime} \boldsymbol{W}_{1}^{\boldsymbol{K}}\right)
$$

is maximized. The decision tree associated with the two-period portfolio selection problem is shown in Figure 2.

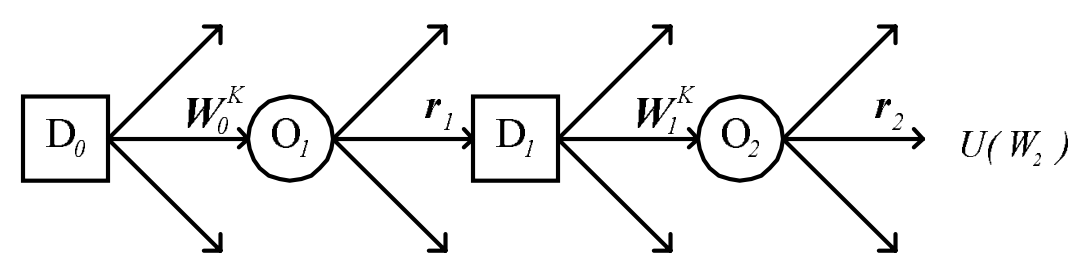

Figure 2. Decision tree for the two-period portfolio selection problem. 
We assume that at time period $t$ the $K$ dimensional return vector $\boldsymbol{r}_{t}$ is normally distributed with mean vector $\boldsymbol{\mu}$ and covariance matrix $\boldsymbol{\Sigma}$. We will denote this distribution as

$$
\left(\boldsymbol{r}_{t} \mid \boldsymbol{\mu}, \boldsymbol{\Sigma}\right) \sim \boldsymbol{N}(\boldsymbol{\mu}, \boldsymbol{\Sigma})
$$

and following Winkler and Barry (1975) we will assume that $\boldsymbol{r}_{t}$ 's are independent random vectors. For our development in this section we also assume that $\boldsymbol{\mu}$ is unknown and uncertainty about $\boldsymbol{\mu}$ is described by a normal prior

$$
\left(\mu \mid m_{0}, C_{0}\right) \sim N\left(m_{0}, C_{0}\right)
$$

where $\boldsymbol{m}_{0}$ and $C_{0}$ are specified quantities. The covariance matrix $\boldsymbol{\Sigma}$ is assumed to be known in this section.

The two-period decision problem shown in Figure 2 is solved in the conventional manner by folding back the decision tree via taking expectations at the random nodes and maximizing the expected utility at the decision nodes. We assume that the investor has an exponential utility function

$$
U\left(W_{2}\right)=-\exp \left(-A W_{2}\right)
$$

where $A>0$ and $1 / A$ is referred to as the "risk tolerance" and describes the degree of risk aversion. Smaller the $A$ is the higher the risk tolerance is for the investor.

At the random node $O_{2}$ the expected utility $E_{1}\left[U\left(W_{2}\right)\right]$ is evaluated by using the probability distribution of $\boldsymbol{r}_{2}$ given $\boldsymbol{r}_{1}$, that is by using $p\left(\boldsymbol{r}_{2} \mid \boldsymbol{r}_{1}\right)$. Given the above setup, via standard Bayesian updating it can be shown that

$$
\left(r_{2} \mid r_{1}\right) \sim N\left(m_{1}, C_{1}+\Sigma\right)
$$

where

$$
\boldsymbol{m}_{1}=C_{1}^{-1}\left(C_{0}^{-1} \boldsymbol{m}_{0}+\boldsymbol{\Sigma}^{-1} \boldsymbol{r}_{1}\right) \text { and } C_{1}=\left(C_{0}^{-1}+\boldsymbol{\Sigma}^{-1}\right)^{-1}
$$

Since $W_{2}=W_{1}+\boldsymbol{r}_{2}^{\prime} \boldsymbol{W}_{1}^{K}$, given $\boldsymbol{r}_{1}, W_{2}$ will also have a normal distribution. Thus, at random node $O_{2}$ the expected utility $E_{1}\left[U\left(W_{2}\right)\right]$ is obtained as

$$
E_{1}\left[U\left(W_{2}\right)\right]=-\int \exp \left(-A W_{2}\right) p\left(W_{2} \mid \boldsymbol{r}_{1}\right) d W_{2}
$$

Using the properties of normal distribution it can be shown that 


$$
E_{1}\left[U\left(W_{2}\right)\right]=-\exp \left\{-A\left[\left(W_{1}+\boldsymbol{m}_{1}^{\prime} \boldsymbol{W}_{1}^{K}\right)-\frac{A}{2}\left(\left(W_{1}^{K}\right)^{\prime} \boldsymbol{P}_{1} \boldsymbol{W}_{1}^{K}\right)\right]\right\}
$$

where $P_{1}=C_{1}+\boldsymbol{\Sigma}$. The optimal portfolio for period two is obtained as

$$
W_{1}^{K *}=\frac{P_{1}^{-1} m_{1}}{A}
$$

by maximizing (2.16) with respect to $W_{1}^{K}$ at decision node $D_{1}$. Optimal value of the expected utility, $U_{1}^{*}\left(W_{2}\right)$, is obtained by evaluating (2.16) using $W_{1}^{K}=W_{1}^{K *}$. Next at random node $O_{1}, E_{0}\left[U_{1}^{*}\left(W_{2}\right)\right]$ needs to be obtained using the predictive distribution $p\left(\boldsymbol{r}_{1}\right)$ which is a normal density with mean vector $\boldsymbol{m}_{0}$ and covariance matrix $\left(\boldsymbol{C}_{\mathbf{0}}+\boldsymbol{\Sigma}\right)$. Note that $U_{1}^{*}\left(W_{2}\right)$ involves $\boldsymbol{m}_{1}$ and $W_{1}$ which both are functions of $\boldsymbol{r}_{1}$ and thus the expectation $E_{0}\left[U_{1}^{*}\left(W_{2}\right)\right]$ can not be evaluated in closed form and the optimal portfolio can not be obtained analytically. In this case one alternative approach is to use a Monte Carlo method to evaluate the expectation and then find the portfolio. But this is not easy to implement for problems with $\mathrm{T}>2$ periods and maybecome computationally inefficient in the multivariate random variance models that are considered in section 3 .

\section{Multivariate Random Variance Models}

In this section we assume that the conditional variance of returns is a function of time, that is,

$$
\left(\boldsymbol{r}_{t} \mid \boldsymbol{\mu}, \boldsymbol{\Sigma}_{\mathbf{t}}, \mathbf{D}_{t-1}\right) \sim N\left(\mu, \boldsymbol{\Sigma}_{\mathbf{t}}\right)
$$

where $\mathbf{D}_{t-1}=\left(\boldsymbol{r}_{1}, \boldsymbol{r}_{2}, \ldots, \boldsymbol{r}_{t-1}\right)$ and we treat $\boldsymbol{\Sigma}_{\mathbf{t}}$ as a stochastic process. In so doing, we will consider two classes of models. The first model is a state-space type stochastic volatilility model which has been considered by Uhlig (1997) and Liu (2000) and the second model is the multivariate GARCH model of Bollerslev et. al. (1988). As previously mentioned other types of stochastic processes such as discrete time gamma processes can also be considered to model conditional variances. In what follows we first introduce the models and then consider the portfolio selection problem using these models and discuss computational issues.

\subsection{State Space Volatility Model}

We first rewrite (3.1) as a linear model with observation noise $\xi_{t}$ as

$$
\boldsymbol{r}_{t}=\boldsymbol{\mu}_{\mathrm{t}}+\boldsymbol{\xi}_{t}
$$


where $\boldsymbol{\xi}_{t} \mid \mathbf{D}_{t-1} \sim \boldsymbol{N}\left(\mathbf{0}, \boldsymbol{\Phi}_{t}^{-1}\right)$ and $\boldsymbol{\Phi}_{t}=\boldsymbol{\Sigma}_{\mathbf{t}}^{-1}$ is the $K \times K$ precision matrix. We will assume that the prior for mean return vector $\boldsymbol{\mu}_{\mathrm{t}}$ is given by

$$
\boldsymbol{\mu}_{\mathrm{t}} \mid \boldsymbol{\Phi}_{t}, \mathbf{D}_{t-1} \sim \boldsymbol{N}\left(\boldsymbol{m}_{t-1}, \boldsymbol{\Phi}_{t}^{-1} / v\right)
$$

where $v$ is a specified precision parameter and $\boldsymbol{m}_{t-1}$ is the known mean vector at time $t-1$. Conditional on $\boldsymbol{\Phi}_{t}$ 's, $\boldsymbol{\mu}_{\mathrm{t}}$ 's are independent random quantities in (3.3). Following Uhlig (1997) and Liu (2000) the precision matrix $\boldsymbol{\Phi}_{t}$ is assumed to follow a first-order Markov structure as

$$
\boldsymbol{\Phi}_{t}=\mathrm{C}\left(\boldsymbol{\Phi}_{t-1}\right)^{\prime} \boldsymbol{\beta}_{t} \mathrm{C}\left(\boldsymbol{\Phi}_{t-1}\right) / \delta
$$

where $\mathrm{C}\left(\boldsymbol{\Phi}_{t-1}\right)$ is the upper Cholesky decomposition of $\boldsymbol{\Phi}_{t-1}$. Given $\mathbf{D}_{t-1}$ we assume that $\boldsymbol{\beta}_{t}$ is will have a matrix beta distribution [see Uhlig (1994) for definition] denoted as

$$
\boldsymbol{\beta}_{t} \mid \mathbf{D}_{t-1} \sim M \operatorname{Beta}\left(\delta n_{t-1} / 2,(1-\delta) n_{t-1} / 2\right)
$$

and that $\boldsymbol{\Phi}_{t-1}$ has a Wishart distribution [see Uhlig (1994)] with scale matrix $\boldsymbol{P}_{t-1}$ and degrees of freedom $n_{t-1}$ denoted as

$$
\boldsymbol{\Phi}_{t-1} \mid \mathbf{D}_{t-1} \sim W i s h\left(\boldsymbol{P}_{t-1}, n_{t-1}\right)
$$

We note that in the above $0<\delta<1$ where $\delta$ is typically larger than 0.9 and $\delta n_{t-1}>K$ for the matrices to be positive definite. It can be shown that (3.4)-(3.6) imply a prior for $\boldsymbol{\Phi}_{t}$ as

$$
\boldsymbol{\Phi}_{t} \mid \mathbf{D}_{t-1} \sim W i s h\left(\delta \boldsymbol{P}_{t-1}, \delta n_{t-1}\right)
$$

The above development yields a random walk type of evolution for the precision matrix from one period to the next. We note that (3.6) and (3.7) imply that

$$
E\left(\boldsymbol{\Phi}_{t-1} \mid \mathbf{D}_{t-1}\right)=E\left(\boldsymbol{\Phi}_{t} \mid \mathbf{D}_{t-1}\right)=\boldsymbol{P}_{t-1}^{-1} n_{t-1}
$$

that is, the means are the same, but the dispersion increases as we move from period $(t-1)$ to $t$. More specifically, $\boldsymbol{P}_{t-1}$, the scale matrix of $\boldsymbol{\Phi}_{t-1}$, changes to $\delta \boldsymbol{P}_{t-1}$ in (3.7). 
An attractive feature of the above model is that if we start with a prior $\boldsymbol{\Phi}_{0} \mid \mathbf{D}_{0} \sim W i \operatorname{sh}\left(\boldsymbol{P}_{0}, n_{0}\right)$ at time 0 , then a conjugate Bayesian analysis is available. It can be shown that

$$
\boldsymbol{\Phi}_{t} \mid \mathbf{D}_{t} \sim W i s h\left(\boldsymbol{P}_{t}, n_{t}\right)
$$

and

$$
\boldsymbol{\mu}_{\mathrm{t}} \mid \boldsymbol{\Phi}_{t}, \mathbf{D}_{t} \sim \boldsymbol{N}\left(\boldsymbol{m}_{t}, \boldsymbol{\Phi}_{t}^{-1} /(1+v)\right)
$$

where $n_{t}=\delta n_{t-1}+1$,

$$
\boldsymbol{P}_{t}=\delta \boldsymbol{P}_{t-1}+\frac{v}{1+v}\left(\boldsymbol{r}_{t}-\boldsymbol{m}_{t-1}\right)^{\prime}\left(\boldsymbol{r}_{t}-\boldsymbol{m}_{t-1}\right)
$$

and

$$
\boldsymbol{m}_{t}=\frac{1}{1+v} \boldsymbol{r}_{t}+\frac{v}{1+v} \boldsymbol{m}_{t-1}
$$

\subsection{Multivariate GARCH Models}

A multivariate extension of the univariate $\mathrm{ARCH}$ and generalized $\mathrm{ARCH}$ models of Engle (1982) and Bollerslev (1986), is introduced by Bollerslev et. al. (1988). Using the observation model

$$
\boldsymbol{r}_{t}=\boldsymbol{\mu}+\boldsymbol{\xi}_{t}
$$

where $\boldsymbol{\xi}_{t} \mid \mathbf{D}_{t-1} \sim \boldsymbol{N}\left(\mathbf{0}, \boldsymbol{\Sigma}_{t}\right)$, the multivariate $\operatorname{GARCH}(q, p)$ model is defined as

$$
\operatorname{vech}\left(\boldsymbol{\Sigma}_{t}\right)=\boldsymbol{A}_{0}+\sum_{i=1}^{q} \boldsymbol{A}_{i} \operatorname{vech}\left(\boldsymbol{\xi}_{t-i} \boldsymbol{\xi}_{t-i}^{\prime}\right)+\sum_{j=1}^{p} \boldsymbol{B}_{j} \operatorname{vech}\left(\boldsymbol{\Sigma}_{t-j}\right)
$$

In (3.12) vech $(\cdot)$ denotes the column stacking operator of the lower portion of a symmetric matrix, $\boldsymbol{A}_{0}$ is a $s=1 / 2 \times K(K+1)$ vector, $\boldsymbol{A}_{i}$ 's and $\boldsymbol{B}_{j}$ are $s \times s$ matrices. We note that certain conditions need to be satisfied in (3.12) for $\boldsymbol{\Sigma}_{t}$ to be a positive definite matrix. In what follows, we will consider the ARCH models in (3.12), that is, the case where $p=0$. Similar to the univariate ARCH models, multivariate $\operatorname{ARCH}(q)$ models imply that vech $\left(\xi_{t} \xi_{t}^{\prime}\right)$ follows a vector autoregressive process of order $q$. In other words, we can motivate an $\operatorname{ARCH}(q)$ process via 


$$
\operatorname{vech}\left(\boldsymbol{\xi}_{t} \boldsymbol{\xi}_{t}^{\prime}\right)=\boldsymbol{A}_{0}+\sum_{i=1}^{q} \boldsymbol{A}_{i} \operatorname{vech}\left(\boldsymbol{\xi}_{t-i} \boldsymbol{\xi}_{t-i}^{\prime}\right)+\boldsymbol{u}_{t}
$$

where $\boldsymbol{u}_{t}=\operatorname{vech}\left(\boldsymbol{\Sigma}_{t}\right)-\operatorname{vech}\left(\boldsymbol{\xi}_{t} \boldsymbol{\xi}_{t}^{\prime}\right)$ is a zero-mean white-noise process.

We note that the model (3.12) is highly parameterized. For example, for $K=2$, the first order ARCH model can be written as

$$
\left(\begin{array}{c}
\Sigma_{11 \mathrm{t}} \\
\Sigma_{12 \mathrm{t}} \\
\Sigma_{22 \mathrm{t}}
\end{array}\right)=\left[\begin{array}{c}
\alpha_{01} \\
\alpha_{02} \\
\alpha_{03}
\end{array}\right]+\left[\begin{array}{lll}
\alpha_{11} & \alpha_{21} & \alpha_{31} \\
\alpha_{12} & \alpha_{22} & \alpha_{32} \\
\alpha_{13} & \alpha_{23} & \alpha_{33}
\end{array}\right]\left[\begin{array}{c}
\xi_{1, t-1}^{2} \\
\xi_{1, t-1} \cdot \xi_{2, t-1} \\
\xi_{2, t-1}^{2}
\end{array}\right]
$$

A natural simplification is obtained by assuming a diagonal structure for the $A_{1}$ matrix in (3.14), that is, $\alpha_{\mathrm{ij}}=0$ if $\mathrm{i} \neq \mathrm{j}$. As pointed out by Bollerslev et. al. (1988), this simply implies that each covariance depends only on its past values, that is,

$$
\left(\begin{array}{c}
\Sigma_{11 \mathrm{t}} \\
\Sigma_{12 \mathrm{t}} \\
\Sigma_{22 \mathrm{t}}
\end{array}\right)=\left[\begin{array}{c}
\alpha_{01}+\alpha_{11} \xi_{1, t-1}^{2} \\
\alpha_{02}+\alpha_{22} \xi_{1, t-1} \cdot \xi_{2, t-1} \\
\alpha_{03}+\alpha_{33} \xi_{2, t-1}^{2}
\end{array}\right]
$$

Similar simplifications can be obtained for multivariate GARCH models which are motivated by vech $\left(\boldsymbol{\xi}_{t} \xi_{t}^{\prime}\right)$ being a vector ARMA process.

A Bayesian analysis of the multivariate GARCH model using any form of prior distributions for $\boldsymbol{\mu}, \boldsymbol{A}_{0}, \boldsymbol{A}_{i}$ 's and $\boldsymbol{B}_{j}$ 's, requires use of Markov chain Monte Carlo (MCMC) methods. The MCMC approach introduced by Muller and Pole (1998) for univariate GARCH models has been extended to multivariate GARCH models in Soyer and Tanyeri (2004).

\subsection{Portfolio Selection using Random Variance Models}

The two-period portfolio selection problem presented in Section 2.1 requires evaluation of expected utility at random nodes $O_{1}$ and $O_{2}$. As we have pointed out earlier, the expectation at node $\mathrm{O}_{2}$ is with respect to the posterior predictive distribution $p\left(\boldsymbol{r}_{2} \mid \boldsymbol{r}_{1}\right)$. In Section 2.1 where this distribution was given as a normal density in (2.13), we were able to evaluate the expected utility in (2.15).

In the state space volatility model, based on the posterior results (3.9) and (3.10) it is again possible to obtain the posterior predictive distribution $p\left(\boldsymbol{r}_{2} \mid \boldsymbol{r}_{1}\right)$. In 
this case $p\left(\boldsymbol{r}_{2} \mid \boldsymbol{r}_{1}\right)$ is a multivariate-t density with degrees of freedom $\left(\delta n_{1}-K+1\right)$, mean vector $\boldsymbol{m}_{1}$, and scale matrix $\boldsymbol{P}_{1}$. This also implies that $W_{2}$ given $\boldsymbol{r}_{1}$ has a multivariate-t distribution and as a result the expectation in (2.15) can not be evaluated in closed form and thus the optimal portfolio at decision node $D_{1}$ can not obtained analytically.

In the multivariate GARCH models, the posterior analysis can not be obtained analytically and as a result the exact form of the posterior predictive distribution $p\left(\boldsymbol{r}_{2} \mid \boldsymbol{r}_{1}\right)$ is not known. Using MCMC methods we can draw samples from the posterior predictive distribution, but this does not allow us to solve the expectation in (2.15) analytically. Thus, in both of these cases solution of the optimal portfolio selection problem requires use of Monte Carlo methods. In the sequel, we will present Monte Carlo-based methods to solve the multi-period portfolio selection problem with random variances.

\section{Monte Carlo Based Approaches for Portfolio Selection Problem}

We consider the two-period problem presented in Section 2.1 with the conditional variance is described by the random variance models of Section 3 and illustrate how the decision problem can be solved using Monte Carlo based methods. In so doing, we first present a standard Monte Carlo approach and illustrate how this method becomes computationally inefficient in Section 4.1. To alleviate the computational problems, in Section 4.2 we introduce an alternate Monte Carlo method to solve the two-period problem by adopting the simulation based approach of Muller and Parmigiani (1995) where the preposterior analysis is replaced by a surface fitting approach. We discuss extension of the proposed approach to multi-period portfolio selection problems in general as well as the related computational issues.

\subsection{Standard Monte Carlo Approach}

Let $\Theta_{t}$ denote unknown parameters of the model for the returns at time $t$ which can be either described by the state space model of Section 3.1 or by the multivariate GARCH model of Section 3.2. The standard Monte Carlo solution of the decision tree given in Figure 2 involves Monte Carlo averages at random nodes $O_{1}$ and $\mathrm{O}_{2}$ and maximization of these Monte Carlo averages at random nodes $D_{0}$ and $D_{1}$. Folding back the decison tree using the Monte Carlo approach consists of the following steps: 
Step 1: Choose initial wealth $W_{0}$ and portfolio $W_{0}^{K}$. Also, choose the vector of weights $\boldsymbol{w}_{1}^{j s}=\left(w_{1}^{1 s}, w_{1}^{2 s}, \ldots, w_{1}^{K s}\right)$, for $j=1, \ldots, J$, where $w_{1}^{k}$ represents the portion of the wealth $W_{1}$ which will be allocated to security $k$ in period 2's portfolio. Note that $\sum_{k=1}^{K} w_{1}^{k}=1$.

Step 2: Generate $\Theta_{1}^{i}$ and $\boldsymbol{r}_{1}^{i}, i=1, \ldots, I$, based on the prior $p\left(\Theta_{1}\right)$ and prior predictive distribution $p\left(\boldsymbol{r}_{1} \mid \Theta_{1}\right)$ for the model. Compute $W_{1}^{i}=W_{0}+\boldsymbol{r}_{1}^{i \prime} \boldsymbol{W}_{0}^{\boldsymbol{K}}$ and for each $i=1, \ldots, I$, using the weight vector $\boldsymbol{w}_{1}^{j}$, obtain the portfolios $W_{1}^{K j}(i)=w_{1}^{j} W_{1}^{i}$ for $j=1, \ldots, J$.

Step 3: For each $\Theta_{1}^{i}$ and $\boldsymbol{r}_{1}^{i}$, generate $\Theta_{2}^{l}$ and $\boldsymbol{r}_{2}^{l}, l=1, \ldots, L$, based on the model. Compute the utility $U\left(W_{2}^{l}\right)$ where $W_{2}^{l}=W_{1}^{j}+\boldsymbol{r}_{2}^{l \prime} W_{1}^{K j}(i)$ for $l=1$, $\ldots, L, j=1, \ldots, J$ and $i=1, \ldots, I$.

Step 4: At random node $\mathrm{O}_{2}$ evaluate the expectation using the Monte Carlo average

$$
E\left[U\left(W_{2}\right) \mid \boldsymbol{W}_{0}^{K}, \boldsymbol{r}_{1}^{i}, \boldsymbol{W}_{1}^{K \boldsymbol{j}}(i)\right]=\frac{1}{L} \sum_{l=1}^{L} U\left(W_{2}^{l}\right),
$$

for $l=1, \ldots, L, j=1, \ldots, J$ and $i=1, \ldots, I$.

Step 5: At decision node $D_{1}$ optimize (4.1) with respect to $W_{1}^{K}(i)$, that is, find $\boldsymbol{W}_{1}^{K *}(i)$ that maximizes $E\left[U\left(W_{2}\right) \mid \boldsymbol{W}_{0}^{\boldsymbol{K}}, \boldsymbol{r}_{1}^{i}, \boldsymbol{W}_{1}^{K j}(i)\right], j=1, \ldots, J$, and obtain

$$
U_{1}^{*}\left(W_{2}\right)=E\left[U\left(W_{2}\right) \mid \boldsymbol{W}_{0}^{K}, \boldsymbol{r}_{1}^{i}, \boldsymbol{W}_{1}^{K *}(i)\right]
$$

where $W_{1}^{K *}(i)$ is a function of $\boldsymbol{r}_{1}^{i}$.

Step 6: At random node $O_{1}$ compute the preposterior expected utility using the Monte Carlo average

$$
E\left[U_{1}^{*}\left(W_{2}\right) \mid \boldsymbol{W}_{0}^{K}\right]=\frac{1}{I} \sum_{i=1}^{L} E\left[U\left(W_{2}\right) \mid \boldsymbol{W}_{0}^{K}, \boldsymbol{r}_{1}^{i}, \boldsymbol{W}_{1}^{K *}(i)\right]
$$

Step 7: Go to Step 1 and repeat steps 2-6 for a different $W_{0}^{K}$ and finally at decision node $D_{0}$ find the maximizer of $E\left[U_{1}^{*}\left(W_{2}\right) \mid \boldsymbol{W}_{0}^{K}\right]$ which gives us the optimal first period portfolio. 
For implementation of the Monte Carlo approach, in the state space model of Section 3.1 we have $\Theta_{\mathbf{t}}=\left(\boldsymbol{\mu}_{t}, \boldsymbol{\Phi}_{t}\right)$ and in the multivariate GARCH model $\boldsymbol{\Theta}_{\mathbf{t}}=(\boldsymbol{\mu}$, $\boldsymbol{\Sigma}_{\mathrm{t}}$ ) where $\boldsymbol{\Sigma}_{\mathrm{t}}$ is a function of $\boldsymbol{A}_{0}, \boldsymbol{A}_{i}$ 's and $\boldsymbol{B}_{j}$ 's as defined in (3.12). In the state space model initially the prior of $\boldsymbol{\Phi}_{0}$ is specified and $\boldsymbol{\Phi}_{1}$ is generated using (3.7) and given $\Phi_{1}$ with specified value of $\boldsymbol{m}_{0}$ the mean vector $\boldsymbol{\mu}_{1}$ is generated using (3.3) and the return vector $\boldsymbol{r}_{1}$ is generated from (3.2). In step 3 given $\boldsymbol{\Phi}_{1}, \boldsymbol{\Phi}_{2}$ is generated using the transition model (3.4) with $\boldsymbol{\beta}_{2}$ is drawn from (3.5) for Given $\boldsymbol{\Phi}_{2}$ and $\boldsymbol{r}_{1}, \boldsymbol{\mu}_{\mathbf{2}}$ is drawn from (3.3) with $\boldsymbol{m}_{1}$ is updated accordingly. In the multivariate GARCH model, if we assume that we have a multivariate $\operatorname{GARCH}(1,1)$ model then the prior $p\left(\boldsymbol{\mu}, \boldsymbol{A}_{0}, \boldsymbol{A}_{1}\right.$, $\left.\boldsymbol{B}_{1}\right)$ is specified. In so doing, initially we can choose vech $\left(\boldsymbol{\Sigma}_{0}\right)=\boldsymbol{A}_{0}$ and assume that $\boldsymbol{\xi}_{0}=\mathbf{0}$ where $\mathbf{0}$ is a $K$ dimensional vector of zeros. Note that in choosing the prior of $A_{0}, A_{1}, B_{1}$ we need to ensure the positive definiteness of the $\Sigma_{1}$ matrix. Once $\mu$, $\boldsymbol{A}_{0}, \boldsymbol{A}_{1}$ and $\boldsymbol{B}_{1}$ are generated, then $\boldsymbol{\Sigma}_{1}$ is drawn from (3.12) Next the error $\boldsymbol{\xi}_{1}$ is drawn and the return vector $\boldsymbol{r}_{1}$ is generated from (3.11). Given $\boldsymbol{\Sigma}_{1}$ and $\boldsymbol{\xi}_{1}$, for the second period, $\boldsymbol{\Sigma}_{2}$ is drawn from (3.12) and $\boldsymbol{r}_{2}$ is drawn from (3.11).

Note that even for the two-period problem, the implementation of the above Monte Carlo approach is not computationally efficient. The approach requires, for each first period portfolio $W_{0}^{K}$, specification of $J$ second period portfolios $W_{1}^{K}$ and $I \times L$ draws from the returns for each portfolio combination. Thus, evaluation of the Monte Carlo integral (4.1) requires a large computational effort in obtaining $U\left(W_{2}\right)$ for each $W_{0}^{K}$.

\subsection{Surface Fitting Approach}

The computational burden involved in the use of standard Monte Carlo approach is common to many Bayesian decision problems such as the optimal design problems. As pointed out by Mueller (1999), such a large scale simulation for each value of the decision variable fails to exploit the continuity of the expected utility surface. To avoid the computational burden in use of the standard Monte Carlo approach, we will adopt the curve/surface fitting method proposed by Muller and Parmigiani (1995) to the portfolio selection problem. This approach facilitates preposterior analysis by replacing the integration steps required at random nodes of the decision tree by smoothing steps. The maximization steps at the decision nodes are replaced by the maximization of the fitted smooth surfaces.

The proposed approach consists of the following steps:

Step 1: Choose initial wealth $W_{0}$ and portfolio $W_{0}^{K s}$. Also, choose the vector of weights $\boldsymbol{w}_{1}^{s}=\left(w_{1}^{1 s}, w_{1}^{2 s}, \ldots, w_{1}^{K s}\right)$, for $s=1, \ldots, S$, where $\sum_{k=1}^{K} w_{1}^{k}=1$. 
Step 2: Generate $\left(\boldsymbol{\Theta}_{1}^{s}\right.$ and $\left.\boldsymbol{r}_{1}^{\boldsymbol{s}}\right), s=1, \ldots, S$, using $p\left(\boldsymbol{\Theta}_{1}\right)$ and $p\left(\boldsymbol{r}_{1} \mid \boldsymbol{\Theta}_{1}\right)$ for the model. Compute $W_{1}^{s}=W_{0}+\boldsymbol{r}_{1}^{s \prime} W_{0}^{K s}$ and obtain the portfolios $W_{1}^{K s}=\boldsymbol{w}_{1}^{s} W_{1}^{s}$.

Step 3: Given $\left(\Theta_{1}^{s}\right.$ and $\left.\boldsymbol{r}_{1}^{s}\right)$, generate $\Theta_{2}^{s}$ and $\boldsymbol{r}_{2}^{s}, s=1, \ldots, S$, based on the model. Using $S$ points evaluate the utility $U_{2}^{s}=U\left(W_{2}^{s}\right)$, with $W_{2}^{s}=W_{1}^{s}+\boldsymbol{r}_{2}^{s \prime} W_{1}^{K s}$ and record the Monte Carlo sample points $\left(\boldsymbol{W}_{\mathbf{0}}^{K s}\right.$, $\left.W_{1}^{K s}, r_{1}^{s}, r_{2}^{s}\right)$ and $U_{2}^{s}$.

Step 4: Fit a surface $L_{2}\left(W_{0}^{K s}, W_{1}^{K s}, \boldsymbol{r}_{1}^{s}\right)$ to the points $\left(\boldsymbol{W}_{0}^{K s}, \boldsymbol{W}_{1}^{K s}, \boldsymbol{r}_{1}^{s}, U_{2}^{s}\right)$. Note that this step is analogous to taking expectation at random node $\mathrm{O}_{2}$ of the decision tree.

Step 5: Find the maximum of fitted surface $L_{2}\left(W_{0}^{K s}, W_{1}^{K s}, r_{1}^{s}\right)$ over $W_{1}^{K}$ and replace the Monte Carlo points by $\left(\boldsymbol{W}_{\mathbf{0}}^{\boldsymbol{K} \boldsymbol{s}}, \boldsymbol{r}_{1}^{\boldsymbol{s}}, u_{2}^{s}\right)$ where $u_{2}^{s}=L_{2}\left(\boldsymbol{W}_{\mathbf{0}}^{\boldsymbol{K} \boldsymbol{s}}\right.$, $\left.W_{1}^{K *}, r_{1}^{s}\right)$ and $W_{1}^{K *}$ is the maxima of $L_{2}\left(W_{0}^{K s}, W_{1}^{K s}, r_{1}^{s}\right)$. Here $W_{1}^{K *}$ is the solution of the portfolio selection problem for the second period and it depends on $\left(\boldsymbol{W}_{0}^{K s}, \boldsymbol{r}_{1}^{\boldsymbol{s}}\right)$. This is analogous to the maximization step at decision node $D_{1}$ of the decision tree.

Step 6: Fit a surface $L_{1}\left(\boldsymbol{W}_{\mathbf{0}}^{K s}\right)$ to the points $\left(\boldsymbol{W}_{\mathbf{0}}^{K s}, u_{2}^{s}\right)$. This is analogous to the expectation taken at random node $O_{1}$ of the tree.

Step 7: Find the maximum of the fitted surface $L_{1}\left(W_{0}^{K s}\right)$ over $W_{0}^{K s}$. This is analogous to the maximization step at decision node $D_{0}$ of the decision tree and the maxima $W_{0}^{K *}$ is the solution of the portfolio selection problem for the first period.

For the multi-period portfolio selection problems with $T>2$ periods, steps 3 through 5 of the proposed algorithm would be repeated $(T-1)$ times.

In implementation of the surface fitting algorithm, generation of random quantities $\Theta_{1}, \boldsymbol{r}_{1}, \Theta_{2}$, and $\boldsymbol{r}_{2}$ under both models follow along the same lines presented for the standard Monte Carlo approach in Section 4.1. However, the computational effort that is required is a lot more modest for the surface fitting approach. As pointed out by Muller (1999), the surface fitting approach does not use a large scale simulation for each allocation but instead exploits the continuity of the expected utility surface. In Step 4 of the algorithm a nonparametric surface $L_{2}\left(W_{0}^{K s}, W_{1}^{K s}, r_{1}^{s}\right)$ is fitted to the points $\left(\boldsymbol{W}_{0}^{K \boldsymbol{s}}, \boldsymbol{W}_{1}^{K \boldsymbol{s}}, \boldsymbol{r}_{1}^{\boldsymbol{s}}, U_{2}^{s}\right)$. We can think about the points $\left(\boldsymbol{W}_{0}^{K \boldsymbol{s}}, \boldsymbol{W}_{1}^{K \boldsymbol{s}}, \boldsymbol{r}_{1}^{\boldsymbol{s}}, U_{2}^{s}\right)$, 
$s=1, \ldots, S$, as the data of a regression problem with $U_{2}$ as the dependent variable and $\left(W_{0}^{K}, W_{1}^{K}, r_{1}\right)$ as the vector of predictors, or the independent variables. Thus, this step is an approximation to the conditional expectation with respect to $\boldsymbol{r}_{2}$. In Step 5 , we maximize the fitted surface $L_{2}$ with respect to $W_{1}^{K}$, which is a multidimensional optimization. This usually requires numerical techniques since most nonparametric regression surface estimators are not available in closed forms. In Step 6 surface $L_{1}\left(\boldsymbol{W}_{0}^{K s}\right)$ is fitted to the points $\left(\boldsymbol{W}_{0}^{K \boldsymbol{s}}, u_{2}^{s}\right)$ and in Step $7 L_{1}\left(\boldsymbol{W}_{0}^{K}\right)$ is maximized with respect to $W_{0}^{K}$ which is a $K$-dimensional optimization. The optimal solution at the decision node $D_{0}$ of the tree gives us the optimal portfolio for the first period. As previously mentioned, it is important to note that the optimal decision at $D_{1}$ represents the optimal portfolio for the second period and this decision depends on both the first period portfolio and $\boldsymbol{r}_{1}$, the returns outcome of the first period.

The choice of the smoothing technique used in steps 4 and 6 depend on the dimensionality of the problem. Nonparametric regression methods or commonly used smoothing methods can be appropriate. In our implementations in Section 5, we used the local regression model Loess of Chambers and Hastie (1992). In some applications the fitted expected utility surfaces may have tendency to be flat, but it is possible to tighten the expected utility surfaces using power transformations as suggested by Muller (1999).

\section{Illustrations of the Monte Carlo Approach}

We will illustrate implementations of the surface fitting approach using the two multivariate random variance models of Section 3 . In so doing, we will consider a twoperiod portfolio selection problem as in Figure 2 and we assume that there are $K=3$ securities in the portfolio where the third security is a risk-free asset. For illustrative purposes we assume that we do not have any historical data and therefore all the simulations will be based on the prior and prior predictive distributions.

In our illustrations we assume that the initial wealth is $W_{0}=1$ and the return on riskless asset is 0.02 . The risk tolerance parameter is specified as $A=0.9$ in (2.12). We will use the surface fitting approach based on $S=1000$ simulations and portfolios. To see the accuracy of our results we will also solve the problem using a standard Monte Carlo approach based on a large scale simulation of $1000^{4}$ samples.

For the state space model of Section 3.1, we will specify the discount parameter as $\delta=0.9$ and the parameters of the Wishart prior for $\boldsymbol{\Phi}_{0} \mid \mathbf{D}_{0}$ as $n_{0}=1000$ and

$$
\boldsymbol{P}_{0}=\left[\begin{array}{ll}
0.020 & 0.002 \\
0.002 & 0.010
\end{array}\right]
$$


The prior parameters of $\boldsymbol{\mu}_{0}$ are chosen as $\boldsymbol{v}=0.8$ and $\boldsymbol{m}_{0}=\left[\begin{array}{ll}0.05 & 0.05\end{array}\right]^{\prime}$. Note that the choice of these prior parameters ensures the positive definiteness of the precision matrices.

We use a multivariate $\mathrm{ARCH}(1)$ model with a diagonal structure given by (3.14) in our illustration. In so doing, we assume that the components of $\boldsymbol{A}_{0}$ vector are independent gamma distributed random quantities, that is, $\boldsymbol{\alpha}_{0, i} \sim \operatorname{Gamma}(0.75,1)$, $i=1, \ldots, 3$. Similarly, the diagonal matrix $\boldsymbol{A}_{1}$ has independent gamma components, $\boldsymbol{\alpha}_{1, i, i} \sim \operatorname{Gamma}(0.75,1), i=1, \ldots, 3$. The mean vector $\boldsymbol{\mu}$ is assumed to have a normal prior with mean vector $\boldsymbol{m}_{0}=\left[\begin{array}{ll}0.03 & 0.025\end{array}\right]^{\prime}$ and variance covariance matrix $C_{0}=\left[\begin{array}{ll}0.010 & 0.006 \\ 0.006 & 0.010\end{array}\right]$. Furthermore, we assume that apriori $A_{0}, A_{1}$, and $\mu$ are independent of each other and specify $\xi_{0}=\left[\begin{array}{ll}0.0 & 0.0\end{array}\right]^{\prime}$.

Note that since the initial wealth is $W_{0}=1$, we can think of the initial portfolio as the weight vector $\boldsymbol{W}_{0}^{3}=\left(w_{0}^{1}, w_{0}^{2}, w_{0}^{3}\right)$ such that $\sum_{k=1}^{3} w_{0}^{k}=1$. Thus, $w_{0}^{3}$ represents proportion of $W_{0}$ which is allocated to the risk-free asset in the first period's portfolio.

We first present results of implementing the surface fitting algorithm of Section 4.2 with the state space volatility model using a simulation size of $S=1000$ in the algorithm. Based on the above priors the optimal portfolio for period 1 is chosen as $w_{0}^{1 *}=0.352, w_{0}^{2 *}=0.598$ implying that $w_{0}^{3 *}=0.05$. In other words, approximately $35 \%$ of the wealth is allocated to the first security, $65 \%$ is allocated to the second security and remaining $5 \%$ is allocated to the risk-free asset. To obtain the exact optimal portfolio we used the standard Monte Carlo method of Section 4.1 and ran a large scale simulation of $1000^{4}$ samples. This also resulted in an allocation of $w_{0}^{1 *}=0.35$, and $w_{0}^{2 *}=0.60$ implying the accuracy of the results from surface fitting algorithm. The optimal first period portfolio weights for the surface fitting and the standard Monte Carlo approaches are shown in Table 1.

Table 1: Comparison of Optimal Portfolios using the Surface Fitting and Standard Monte Carlo (SMC) Approaches

\begin{tabular}{|c|c|c|c|}
\hline$w_{0}^{1 *}$ & $w_{0}^{2 *}$ & $w_{0}^{1 *}(\mathrm{SMC})$ & $w_{0}^{2 *}(\mathrm{SMC})$ \\
\hline 0.352 & 0.598 & 0.350 & 0.600 \\
\hline
\end{tabular}

In Figure 3, we present the plot of the fitted expected utility surface that we obtain at the random node $O_{1}$ of the decision tree. In other words, this correponds to the fitted surface $L_{1}\left(\boldsymbol{W}_{0}^{2}\right)$ to the points $\left(\boldsymbol{W}_{0}^{2}, u_{2}\right)$ in Step 6 of the algorithm presented in Section 4.2. Note that the optimal portfolio $w_{0}^{1 *}=0.352$ and $w_{0}^{2 *}=0.598$ is 
obtained by maximizing this expected utility surface with respect to $w_{0}^{1}$ and $w_{0}^{2}$ at the decision node $D_{0}$ as implied by the Step 7 of the algorithm.

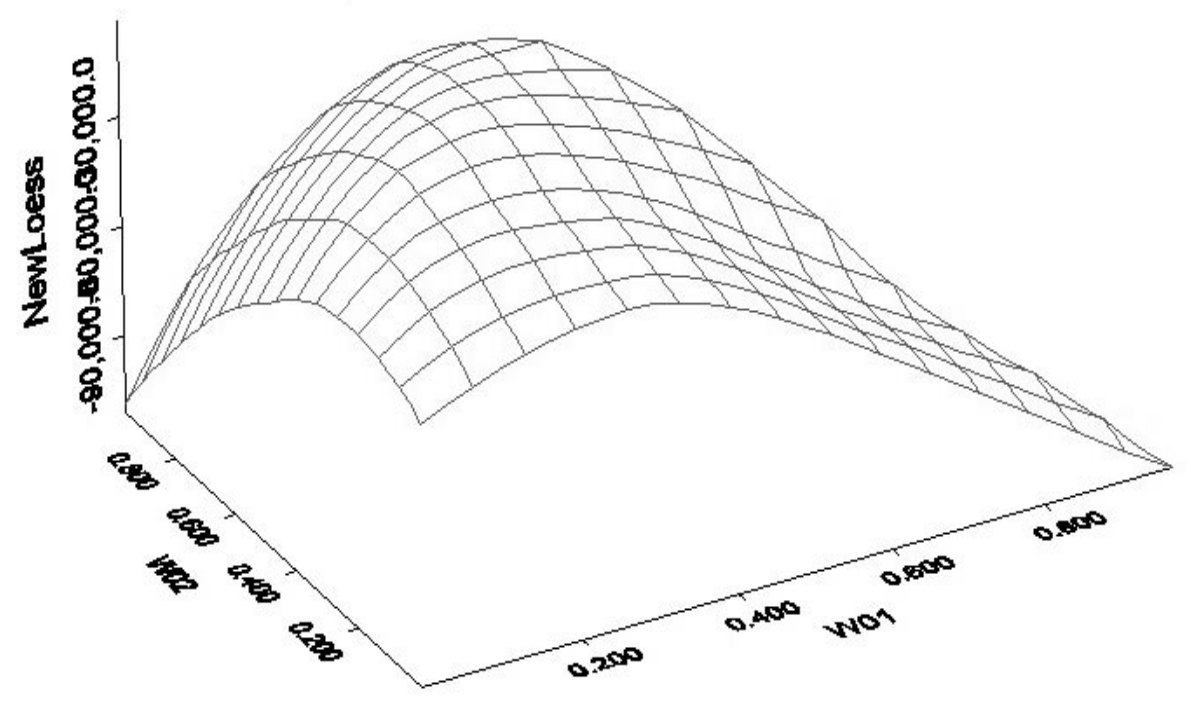

Figure 3. Expected Utility Surface as a Function of $w_{0}^{1}$ and $w_{0}^{2}$.

As we have previously pointed out, the selection of the optimal portfolio for the second period will be made after observing the first period returns. Thus, this selection is a function of both $W_{0}^{3}$, the portfolio of the first period, and the observed return vector $\boldsymbol{r}_{1}$. Two examples of optimal portfolios $W_{1}^{3 *}$ for period two for selected values of $W_{0}^{3}$ and $r_{1}$ are shown in Table 2. Columns 1 and 2 of the table show the selected allocations to the risky securities whereas columns 3 and 4 show the returns observed at period 1. Optimal portfolio allocations for period 2 using the surface fitting approach are illustrated in columns 5 and 6 and the optimal portfolios using the standard Monte Carlo approach are given in columns 7 and 8.

Table 2: Optimal Portfolios for Period 2 as a Function of $\boldsymbol{W}_{0}^{3}$ and $\boldsymbol{r}_{1}$ using the Surface Fitting and Standard Monte Carlo (SMC) Approaches

\begin{tabular}{|c|c|c|c|c|c|c|c|c|}
\hline & $w_{0}^{1}$ & $w_{0}^{2}$ & $r_{1}^{1}$ & $r_{1}^{2}$ & $w_{1}^{1 *}$ & $w_{1}^{2 *}$ & $w_{1}^{1 *}(\mathrm{SMC})$ & $w_{1}^{2 *}(\mathrm{SMC})$ \\
\hline case I & 0.712 & 0.007 & -0.0894 & 0.0970 & 0.002 & 0.963 & 0.000 & 0.960 \\
\hline case II & 0.806 & 0.165 & -0.0552 & 0.0880 & 0.011 & 0.915 & 0.010 & 0.910 \\
\hline
\end{tabular}

When we compare the results of the two approaches, we see that they are very close. Thus, the surface fitting algorithm, which is based on a simulation effort of only 
$S=1000$ samples, seems very accurate. Note that under the first case in the table, a portfiolio of $w_{0}^{1}=0.712$ and $w_{0}^{2}=0.007$ is specied implying that $w_{0}^{3}=0.281$ is allocated to the risk-free asset. During the first period the returns on risky securities are observed as $r_{1}^{1}=-0.0894$ and $r_{1}^{2}=0.0970$. As a result of this loss in security 1 and gain in security 2 , the optimal portfolio for period 2 is revised to $w_{1}^{1^{*}}=0.002$, $w_{1}^{2 *}=0.963$ and $w_{1}^{3 *}=0.035$. Note that since we observe a loss on security 1 and the return on risk-free asset is 0.02 , allocation is shifted to security 2 after the first period.

In Figure 4, we present expected utility surface associated with the case 1 of Table 2. This corresponds to the expectation taken at at the random node $\mathrm{O}_{2}$ of the decision tree and it is obtained as fitting the surface $L_{2}\left(\boldsymbol{W}_{0}^{2}, \boldsymbol{W}_{1}^{2}, \boldsymbol{r}_{1}\right)$ to the points $\left(\boldsymbol{W}_{0}^{2}, \boldsymbol{W}_{1}^{2}, \boldsymbol{r}_{1}, U_{2}\right)$ in Step 4 of the algorithm. Note that the optimal portfolio $w_{1}^{1 *}=0.002$ and $w_{1}^{2 *}=0.963$ is obtained by maximizing this expected utility surface with respect to $w_{1}^{1}$ and $w_{1}^{2}$ at the decision node $D_{1}$ as implied by the Step 5 of the algorithm.

Similar analysis can be done by using the multivariate $\mathrm{ARCH}(1)$ model with a diagonal structure using the priors presented earlier. Note that in this case prior mean return of the first security is higher than the second one. Based on the surface fitting approach with $S=1000$ samples we have obtained the first period portfolio as $w_{0}^{1 *}=0.674, w_{0}^{2 *}=0.321$ implying that $w_{0}^{3 *}=0.005$. Use of the standard Monte Carlo approach with $1000^{4}$ samples resulted in very similar results again implying the accuracy of the surface fitting algorithm. These results are shown in Table 3.

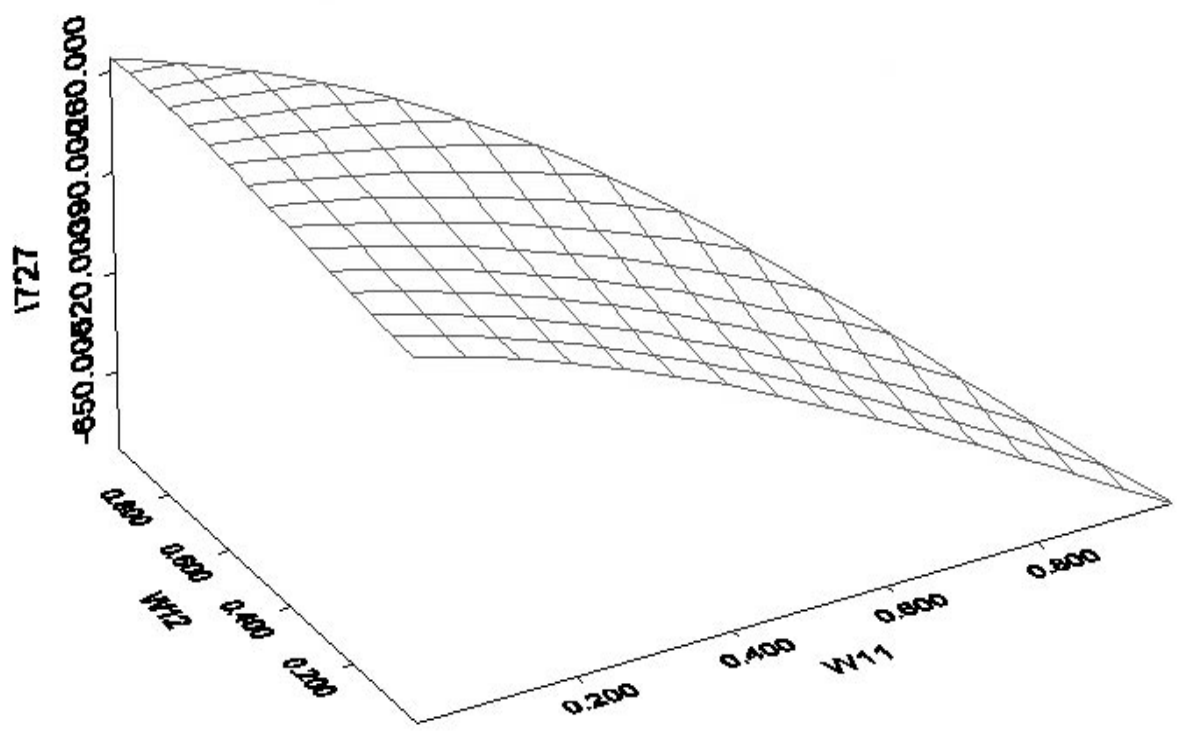

Figure 4. Expected Utility Surface for Case 1 as a Function of $w_{1}^{1}$ and $w_{1}^{2}$. 
Table 3: Optimal Portfolios using the Surface Fitting and Standard

Monte Carlo (SMC) Approaches with ARCH(1) model

\begin{tabular}{|c|c|c|c|}
\hline$w_{0}^{1 *}$ & $w_{0}^{2 *}$ & $w_{0}^{1 *}(\mathrm{SMC})$ & $w_{0}^{2 *}(\mathrm{SMC})$ \\
\hline 0.674 & 0.321 & 0.680 & 0.320 \\
\hline
\end{tabular}

Two examples of optimal portfolios $W_{1}^{3 *}$ for period two for selected values of $W_{0}^{3}$ and $\boldsymbol{r}_{1}$ are shown in Table 4 using the ARCH(1) model. As implied by the comparison with the standard Monte Carlo algorithm results, the surface fitting algorithm looks very accurate. Note that under the first case in the table, a portfiolio of $w_{0}^{1}=0.387$ and $w_{0}^{2}=0.578$ is specied implying that $w_{0}^{3}=0.035$ is allocated to the risk-free asset. As a result of loss in security 2 and gain in security 1 during the first period, the optimal portfolio for period 2 is revised to $w_{1}^{1 *}=0.853, w_{1}^{2 *}=0.000$ and $w_{1}^{3 *}=0.147$.

Table 4: Optimal Portfolios for Period 2 using the Surface Fitting and Standard Monte Carlo (SMC) Approaches ARCH(1) model

\begin{tabular}{|c|c|c|c|c|c|c|c|c|}
\hline & $w_{0}^{1}$ & $w_{0}^{2}$ & $r_{1}^{1}$ & $r_{1}^{2}$ & $w_{1}^{1 *}$ & $w_{1}^{2 *}$ & $w_{1}^{1 *}(\mathrm{SMC})$ & $w_{1}^{2 *}(\mathrm{SMC})$ \\
\hline case I & 0.387 & 0.578 & 0.0209 & -0.0283 & 0.853 & 0.000 & 0.850 & 0.000 \\
\hline case II & 0.303 & 0.576 & -0.0205 & 0.0089 & 0.002 & 0.077 & 0.000 & 0.080 \\
\hline
\end{tabular}

\subsection{Sensitivity Results and Comparison with Single-period Problem}

An important parameter in our implementation of the proposed surface fitting approach is $S$. Note that specification of $S$ determines the decision space, that is, the number of different feasible portfolios as well as the simulation effort. For computational efficiency, it is desirable to obtain accurate results with a small value of $S$. We have invetigated the robustness of the optimal portfolio using different values of $S$ in the examples that are presented here and have found out that the optimal portfolios are very similar and accurate (when compared to standard Monte Carlo results) for $S=500,1000,2000$.

We have also invetigated the sensitivity to prior parameters. It is clear that the optimal allocation for period 1 depends on the choice of the prior parameters in the two multivariate random variance models. Especially, the allocation is expected to be quite sensitive to changes in parameters associated with mean and precision of security returns. To investigate this we have looked at how the optimal allocation in period 1 
changes in the state space model when we change the prior parameters of mean return vector $\boldsymbol{\mu}_{0}$ and the precision matrix $\boldsymbol{\Phi}_{0}$. More specifically we have investigated the sensitivity of the results to changes in $\boldsymbol{m}_{0}$ and $\boldsymbol{P}_{0}$.

In the example that we have presented in the above the prior mean vector is specified as $\boldsymbol{m}_{0}=\left[\begin{array}{ll}0.05 & 0.05\end{array}\right]^{\prime}$ implying equal expected returns for the two risky securities. As expected, keeping all the other prior specifications at the same levels, when we have increased the prior expected return on the first security, optimal allocation to the first security has increased. An example of this is given in Table 5.

Table 5: Sensitivity of Optimal Portfolios to $\boldsymbol{m}_{0}$ in the State Space Model

\begin{tabular}{|c|c|c|c|}
\hline \multicolumn{2}{|c|}{$\boldsymbol{m}_{0}$} & $w_{0}^{1 *}$ & $w_{0}^{2 *}$ \\
\hline$[0.05$ & 0.05 & 0.352 & 0.598 \\
\hline$[0.10$ & $0.05]$ & 0.551 & 0.423 \\
\hline$[0.15$ & 0.05 & 0.629 & 0.194 \\
\hline
\end{tabular}

Similarly, we can look at changes in the prior scale matrix $\boldsymbol{P}_{0}$ for the precision of returns. We note that the properties of the Wishart distribution and (3.8) imply that $E\left(\boldsymbol{\Phi}_{0}^{-1} \mid \mathbf{D}_{0}\right)=E\left(\boldsymbol{\Phi}_{1}^{-1} \mid \mathbf{D}_{0}\right) \propto \boldsymbol{P}_{0}$, that is, the expected conditional variance (or the inverse precision) is proportional to the scale matrix $\boldsymbol{P}_{0}$. Thus, increasing any of the diagonal elements of $\boldsymbol{P}_{0}$ implies that the expected variance of the corresponding security return will increase. As expected this implies a shift in allocation to the less riskier security. We have investigated this by increasing the first security's prior parameter in $\boldsymbol{P}_{0}$ from 0.02 to 0.05 and have observed that the original allocation of $\left(w_{0}^{1 *}=0.352, w_{0}^{2 *}=0.598\right)$ has changed to $\left(w_{0}^{1 *}=0.205, w_{0}^{2 *}=0.677\right)$. In other words, the increase in uncertainty about security 1 return has resulted in a decrease in allocation to this asset. Similar insights can be obtained in the multivariate GARCH model by changing the prior parameters $\boldsymbol{m}_{0}$ and $\boldsymbol{C}_{0}$ of the model.

In addition to sensitivity analysis, we have also investigated how the optimal portfolios obtained using the two-period model differ from those obtained using the single-period model. In so doing, we have compared the optimal portfolios that are obtained for the first period under the two models using the state space volatility model. Note that the single period problem does not take into account any uncertainty associated with the second period returns and thus we expect that the optimal portfolios chosen will be different under the two models. The difference between the two sets of optimal portfolios depends on the degree of prior uncertainty as reflected by the parameters. In our example we have assumed the prior mean return vector $\boldsymbol{m}_{0}=\left[\begin{array}{lll}0.05 & 0.05\end{array}\right]^{\prime}$ and the prior precision scale matrix $\boldsymbol{P}_{0}=\left[\begin{array}{ll}0.020 & 0.002 \\ 0.002 & 0.010\end{array}\right]$ with 
degrees of freedom $n_{0}=1000$. It can be seen from (3.8) that these prior specifications imply a high degree of expected precision for both security returns for periods 1 and 2 . Thus, as a result the optimal portfolios that are obtained under the two models are not expected to differ much. The optimal portfolio under the single period model is obtained as $\left(w_{0}^{1 *}=0.398, w_{0}^{2 *}=0.583\right)$ which is slightly different than the optimal portfolio given in Table 1 for the two-period problem..

When we decrease the precision of the first security by specifying the scale matrix as $\boldsymbol{P}_{0}=\left[\begin{array}{ll}0.050 & 0.002 \\ 0.002 & 0.010\end{array}\right]$, we observe a more pronounced difference between the two portfolios as shown in Table 6. Similarly, changing the prior mean vector significantly yields more different optimal portfolios under the two models. For example, if the prior mean is specified as $\boldsymbol{m}_{0}=\left[\begin{array}{ll}0.15 & 0.05\end{array}\right]^{\prime}$ then the optimal portfolio under the single-period model is obtained as $\left(w_{0}^{1 *}=0.533, w_{0}^{2 *}=0.278\right)$ which is quite different than the portfolio $\left(w_{0}^{1 *}=0.629, w_{0}^{2 *}=0.194\right)$ obtained under the two-period problem as shown in Table 5.

Table 6: Comparison of Optimal Portfolios for the Single and Two-Period Problems

\begin{tabular}{|c|c|c|}
\hline Problem & $w_{0}^{1 *}$ & $w_{0}^{2 *}$ \\
\hline Two-period & 0.205 & 0.677 \\
\hline Single-period & 0.322 & 0.583 \\
\hline
\end{tabular}

\section{REFERENCES}

Aguilar, O., West, M., 2000. Bayesian dynamic factor models and portfolio allocation, Journal of Business and Economic Statistics 18 338-357.

Barry, C. B., Winkler, R. L., 1976. Nonstationarity and portfolio choice, Journal of Financial and Quantitative Analysis 11 217-235.

Bollerslev, T., 1986. Generalized autoregressive conditional heteroskedasticity, Journal of Econometrics 31 307-327.

Bollerslev, T., Engle, R. F., Wooldridge, J. M., 1988. A capital asset pricing model with time-varyimg covariances, Journal of Political Economy 96 116-131.

Chambers, J. M., Hastie, T. J., 1992. Statistical Models in S, Wadsworth. 
Engle, R. F., 1982. Autoregressive conditional heteroskedasticity with estimates of the variance of United Kingdom inflation, Econometrica 50 987-1007.

Farrar, D. E, 1962. The Investment Decision Under Uncertainty, Englewood Cliffs.

Harvey, A., Ruiz, E., Shephard, N., 1994. Multivariate stochastic variance models, Review of Economic Studies 61 247-264.

Jacquier, E., Polson, N. G., Rossi, P., 1994. Bayesian analysis of stochastic volatility models, Journal of Business and Economic Statistics 12 371-388.

Lindley, D. V., 1985. Making Decisions, John Wiley and Sons, New York.

Liu, J., 2000. Bayesian time series analysis using simulation based computation. Unpublished Ph. D. Dissertation, Institute of Statistics and Decision Sciences, Duke University.

Mao, J. C. T., Sarndal, C. E., 1966. A decision theory approach to portfolio selection, Management Science 12 323-333.

Markowitz, H, 1952. Portfolio selection, Journal of Finance 7 77-91.

Merton, R.C., 1973. An intertemporal capital asset pricing model, Econometrica 41 866-887.

Mossin, J., 1968. Optimal multiperiod portfolio policies, Journal of Business 41215 229.

Muller, P., 1999. Simulation based optimal design. In: Bernardo, J. M., Berger, J. O., Dawid, A. P., Smith, A. F. M. (Eds.), Bayesian Statistics 6, Oxford University Press, New York, pp. 459-474.

Muller, P., Parmigiani, G., 1995. Optimal design via curve fitting of Monte Carlo experiments, Journal of the American Statistical Association 90 503-510.

Muller, P., Pole, A., 1998. Monte Carlo posterior integration in GARCH models, Sankhya, Series B 60 127-144.

Polson, N. G., Tew, B. V., 2000. Bayesian portfolio selection: An empirical analysis of the SP500 index 1970-1996, Journal of Business and Economic Statistics 18 164-173.

Quintana, J. M., 1992. Optimal portfolios of forward currency contracts, In: Bernardo, J. M., Berger, J. O., Dawid, A. P., Smith, A. F. M. (Eds.), Bayesian Statistics 4, Oxford University Press, New York, pp. 753-762.

Quintana, J. M., Putnam, B. H., 1994. Driving on hazardous road with limited visibility, Technical Report, Putnam and Associates, USA. 
Quintana, J. M., Putnam, B. H., 1996. Debating currency markets efficiency using multiple-factor models, Proceedings of the ASA Section on Bayesian Statistical Science, American Statistical Association.

Quintana, J. M., Putnam, B. H., Wilford, D. S., 1998. Mutual and pension funds management: Beating the markets using a global Bayesian investment strategy, Proceedings of the ASA Section on Bayesian Statistical Science, American Statistical Association.

Quintana, J. M., Lourdes, V., Aguilar, O., Liu, J., 2003. Global gambling, In: Bernardo, J. M., Bayarri, M. J., Berger, J. O., Dawid, A. P., Heckerman, D., Smith, A. F. M., West, M. (Eds.), Bayesian Statistics 7, Oxford University Press, New York, pp. 349-368.

Soyer, R., Tanyeri, K., 2003. Bayesian analysis of a random environment model for security returns, Technical Report, Department of Management Science, The George Washington University, USA.

Soyer, R., Tanyeri, K., 2004. A Short Note on Bayesian Analysis of Multivariate GARCH Models Returns, Technical Report, Department of Management Science, The George Washington University, USA.

Uhlig, H., 1994. On singular Wishart and singular multivariate beta distribution, Annals of Statistics 22 395-405.

Uhlig, H., 1997. Bayesian vector autoregressions with stochastic volatility, Econometrica 65 59-73.

Winkler, R. L., Barry, C. B., 1975. A Bayesian model for portfolio selection and revision, Journal of Finance 30 179-192. 